Article

\title{
One Pot Single Step Synthesis and Biological Evaluation of Some Novel Bis(1,3,4-thiadiazole) Derivatives as Potential Cytotoxic Agents
}

\author{
Sobhi M. Gomha ${ }^{1}$, Nabila A. Kheder ${ }^{1,2}$, Abdou O. Abdelhamid ${ }^{1}$ and Yahia N. Mabkhot ${ }^{3, *}$ \\ 1 Department of Chemistry, Faculty of Science, Cairo University, Giza 12613, Egypt; \\ s.m.gomha@gmail.com (S.M.G.); nabila.abdelshafy@gmail.com (N.A.K.); \\ abdelhamid45@gmail.com (A.O.A.) \\ 2 Department of Pharmaceutical Chemistry, Faculty of Pharmacy, King Khalid University, \\ Abha 61441, Saudi Arabia \\ 3 Department of Chemistry, College of Science, King Saud University, P.O. Box 2455, \\ Riyadh 11451, Saudi Arabia \\ * Correspondence: yahia@ksu.edu.sa; Tel.: +966-11-467-5898; Fax: +966-11-467-5992
}

Academic Editor: Panayiotis A. Koutentis

Received: 11 October 2016; Accepted: 9 November 2016; Published: 15 November 2016

\begin{abstract}
A novel series of bis(1,3,4-thiadiazole) derivatives were synthesized in one step methodology with good yields by condensation reaction between bis-hydrazonoyl chloride 1 and various reagents. The structures of the prepared compounds were confirmed by spectral data (IR, NMR, and MS), and elemental analysis. The anticancer activity against human breast carcinoma (MCF-7) cancer cell lines was evaluated in MTT assay. The results revealed that the bis-thiadiazole derivatives $\mathbf{5 c}, \mathbf{d}, \mathbf{7 b}, \mathbf{c}$ and $\mathbf{9 c}$ had higher antitumor activity than the standard drug Imatinib.
\end{abstract}

Keywords: bis-hydrazonoyl chlorides; bis(1,3,4-thiadiazole); anticancer activity; methyl arylidene dithiocarbamate; dipolar cycloaddition reaction

\section{Introduction}

Many compounds having a 1,3,4-thiadiazole ring in their skeleton are capable of versatile pharmacological activities [1]. Also, thiadiazoles have been introduced as anticonvulsant [2], anti-parkinsonism [3], anti-histaminic [4] and anti-asthmatic [5], antitumor [6,7], analgesic [8], antimicrobial [9,10], and antitubercular [11-13] agents. Additionally, many drugs containing a 1,3,4-thiadiazole ring are available in the market, such as the carbonic anhydrase inhibitors Acetazolamide and Methazolamide (Figure 1).

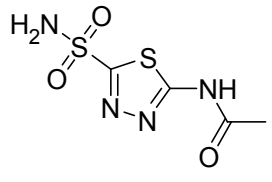

Acetazolamide

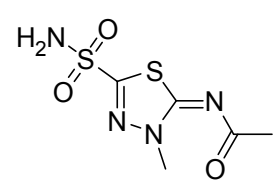

Methazolamide

Figure 1. Examples of medications containing 1,3,4-thiadiazole ring.

Imatinib (Figure 2) is a tyrosine-kinase inhibitor used to treat chronic myelogenous leukemia, gastrointestinal stromal tumors and a number of other malignancies. Doxorubicin (Figure 2) is used to treat some leukemias and Hodgkin's lymphoma, cancers of the bladder, breast, stomach, lung, ovaries, thyroid, soft tissue sarcoma, and multiple myeloma. In view of the medicinal importance 
of thiadiazole ring and as a continuation of our interest in the synthesis of a variety of thiadiazole derivatives for biological evaluation [14-23], we reported in the present study, the synthesis of a series of nine bis(1,3,4-thiadiazole) derivatives and their evaluation against breast cancer cell line (MCF-7) using Imatinib and Doxorubicin as a reference drugs.
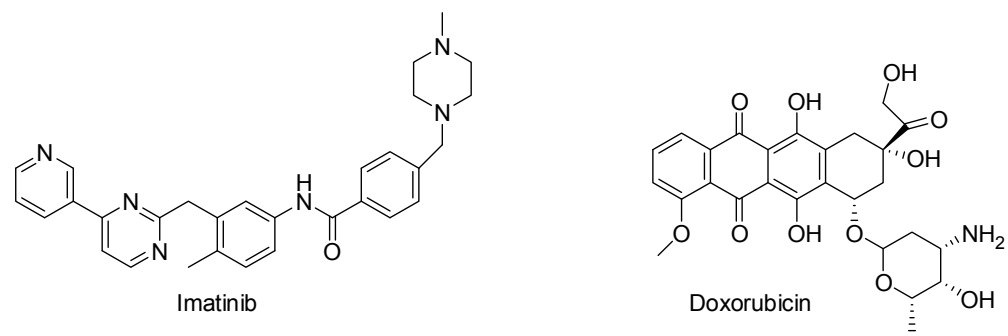

Figure 2. Structure of Imatinib and Doxorubicin.

\section{Results and Discussion}

\subsection{Chemistry}

A mixture of bis-hydrazonoyl chloride 1 [24] and the appropriate methyl arylidene dithiocarbamate 2 [25-28] was stirred at room temperature for $30 \mathrm{~min}$ to afford the corresponding bis(1,3,4-thiadiazole) derivative 5 (Scheme 1). These reactions were assumed to start in each case, via $S$-alkylation with the elimination of two hydrochloric molecules, to afford the non-isolable bis-alkylated intermediate 3, which underwent intramolecular Michael-type addition to give intermediate 4. Elimination of two methanethiol molecules from 4 gave the final product 5 . The structures of the synthesized products $\mathbf{5 a}-\mathbf{d}$ were elucidated based on their spectral (IR, MS, ${ }^{1} \mathrm{H}$ - and ${ }^{13} \mathrm{C}-\mathrm{NMR}$ ) and elemental analyses Their IR spectra showed, in each case, an absorption band in the region $1708-1733 \mathrm{~cm}^{-1}$ due to the carbonyl of the ester group. Also, their ${ }^{1} \mathrm{H}-\mathrm{NMR}$ spectrum exhibited a triplet signals in the region $\delta 1.29-1.32$ and a quartet signals in the region $\delta 4.34-4.35$ due to $\mathrm{CH}_{2}$ and $\mathrm{CH}_{2}$ protons of the ethoxy groups, in addition to the aromatic signals. The mass spectra of the compounds $\mathbf{5 a - d}$ showed, in each case, a peak due to their molecular ions.

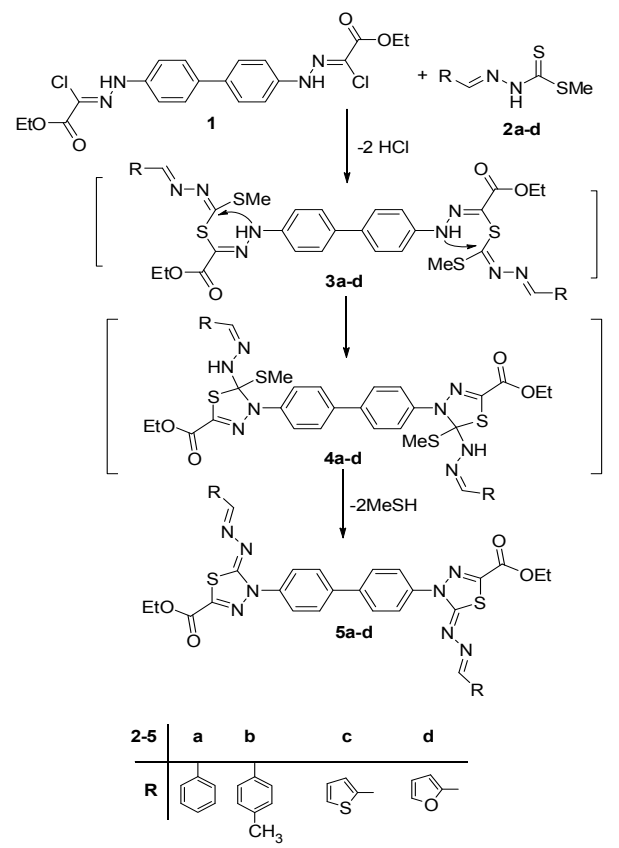

Scheme 1. Synthesis of bis-thiadiazole derivatives $5 \mathbf{a}-\mathbf{d}$. 
Also, the synthesis of combinatorial libraries of bis(1,3,4-thiadiazole) derivatives permits the testing of the biological activities of a vast array of these compounds. So, we intended to repeat the latter experiment again using bis-hydrazonoyl chloride $\mathbf{1}$ with the appropriate methyl arylidene dithiocarbamate $\mathbf{6} \mathbf{a}-\mathbf{c}$ and $\mathbf{8 a}, \mathbf{b}$, under the same experimental conditions, which led to the corresponding bis-thiadiazoles $\mathbf{7} \mathbf{a}-\mathbf{c}$ and $\mathbf{9 a}, \mathbf{b}$, respectively (Schemes 2 and 3). The structures of these products $\mathbf{7 a - c}$ and $\mathbf{9 a}, \mathbf{b}$ were verified by their spectral and elemental analysis.

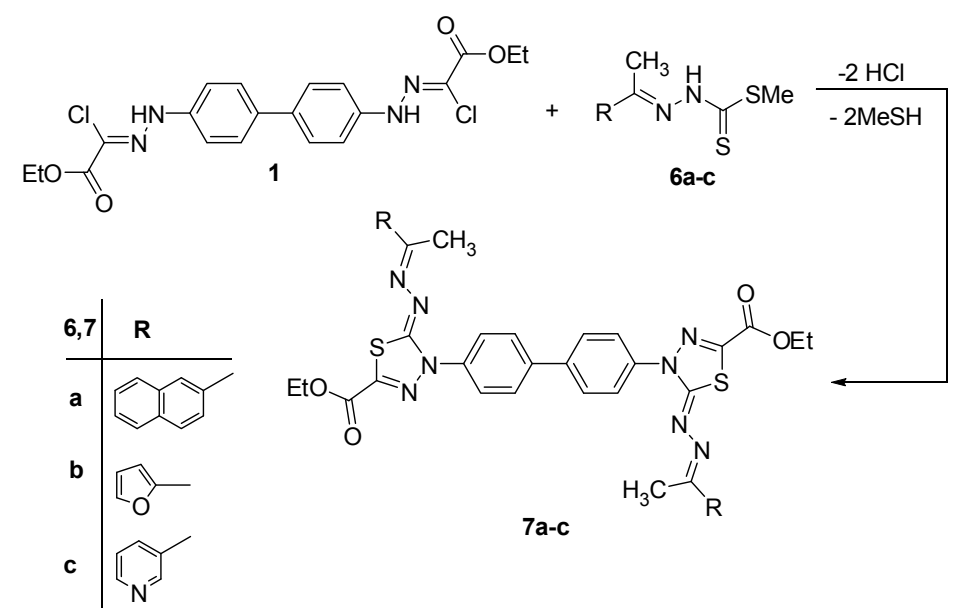

Scheme 2. Synthesis of bis-thiadiazole derivatives $7 \mathbf{a}-\mathbf{c}$.

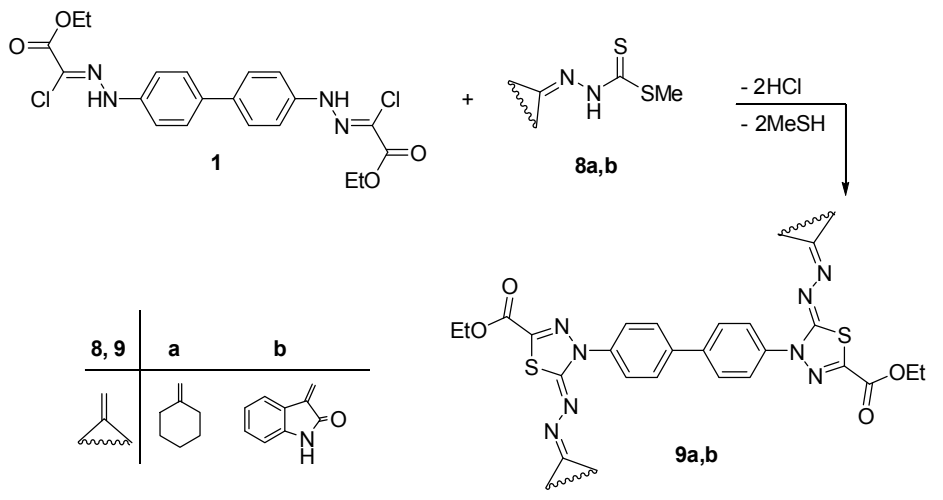

Scheme 3. Synthesis of bis-thiadiazole derivatives $9 \mathbf{a}, \mathbf{b}$.

\subsection{Pharmacology}

Biological Screening of the Synthesized Bis-thiadiazoles for Their Cytotoxic Activity

The in vitro growth inhibitory rates (\%) and inhibitory growth activity (as measured by $\mathrm{IC}_{50}$ ) of the newly synthesized bis-thiadiazoles were determined against human breast carcinoma cell line (MCF-7) in comparison with the well-known anticancer standard drugs doxorubicin and Imatinib $\left(\right.$ Gleevec $\left.^{\circledR}\right)$, using MTT viability assay. Data generated were used to plot a dose-response curve with which the concentration $(\mu \mathrm{M})$ of test compounds required to kill $50 \%$ of the cell population $\left(\mathrm{IC}_{50}\right)$ was determined. Cytotoxic activity was expressed as the mean $\mathrm{IC}_{50}$ of three independent experiments. The difference between inhibitory activities of all bis-thiadiazoles with different concentrations was statistically significant $p<0.001$. Table 1 shows the antitumor activities of the tested bis-thiadiazoles compared with reference standard drugs evaluated using MTT assay on breast cancer cell line (MCF-7).

The previous results lead to the following conclusions. 
- The bis-thiadiazole derivatives $\mathbf{5} \mathbf{c}, \mathbf{d}, \mathbf{7 b}, \mathbf{c}$ and $\mathbf{9 b}$ had higher antitumor activity than the standard drug Imatinib.

- The bis-thiadiazole derivatives $\mathbf{5 a}, \mathbf{b}$ and $\mathbf{9 a}$ have moderate activity.

- The bis-thiadiazole derivative 7a has poor antitumor activity against human breast carcinoma cell line (MCF-7).

- The heterocyclic rings such as pyridine in $\mathbf{7 c}$, thiophene in $\mathbf{5 c}$, furan in $\mathbf{5 d}, \mathbf{7 b}$ and indole in $\mathbf{9 b}$ are necessary to have the higher antitumor activity.

Table 1. The antitumor activities of the tested bis-thiadiazoles $5 \mathbf{a}-\mathbf{d}, \mathbf{7} \mathbf{a}-\mathbf{c}, \mathbf{9 a}, \mathbf{b}$.

\begin{tabular}{|c|c|}
\hline Compd. No. & $\mathrm{IC}_{50}(\mu \mathrm{g} / \mathrm{mL})$ \\
\hline $5 a$ & $45.2 \pm 1.4$ \\
\hline $5 b$ & $37.5 \pm 1.7$ \\
\hline $5 c$ & $18.4 \pm 1.2$ \\
\hline $5 d$ & $20.2 \pm 0.6$ \\
\hline $7 a$ & $145.1 \pm 3.4$ \\
\hline $7 b$ & $21.7 \pm 1.5$ \\
\hline $7 \mathrm{c}$ & $16.9 \pm 1.7$ \\
\hline $9 a$ & $80.0 \pm 2.0$ \\
\hline $9 b$ & $20.7 \pm 2.4$ \\
\hline Doxorubicin & $0.8 \pm 0.1$ \\
\hline Imatinib & $24.5 \pm 0.3$ \\
\hline
\end{tabular}

\section{Materials and Methods}

\subsection{Chemistry}

\subsubsection{General}

Measurements of the melting points were carried out on Electrothermal IA 9000 series digital melting point apparatus (Bibby Sci. Lim. Stone, Staffordshire, UK). The IR spectra were recorded in potassium bromide discs on a Pye Unicam SP 3300 and Shimadzu FT-IR 8101 PC infrared spectrophotometer (Shimadzu, Tokyo, Japan). ${ }^{1} \mathrm{H}-\mathrm{NMR}$ and ${ }^{13} \mathrm{C}-\mathrm{NMR}$ spectra were measured in deuterated dimethyl sulfoxide (DMSO- $d_{6}$ ) using a Varian Gemini 300 NMR spectrometer (Varian, Inc., Karlsruhe, Germany). Mass spectra were recorded on a Shimadzu GCMS-QP1000 EX mass spectrometer (Tokyo, Japan) at $70 \mathrm{eV}$. Measurements of the elemental analysis were carried out at the Microanalytical Centre of Cairo University, Giza, Egypt. All reactions were followed by TLC (Silica gel, Merck, Kenilworth, NJ, USA). The biological evaluation of the products was carried out at the Regional Center for Mycology and Biotechnology at Al-Azhar University, Cairo, Egypt. Bis-hydrazonoyl 
chloride $\mathbf{1}$ [24] and the methyl arylidene dithiocarbamate 2, 6, 8 [25-28] were prepared as described in the literature.

\subsubsection{Synthesis of 1,3,4-Thiadiazoline Derivatives $5 \mathbf{a}-\mathbf{d}, \mathbf{7} \mathbf{a}-\mathbf{c}$ and $\mathbf{9 a}, \mathbf{b}$}

Triethylamine $(0.14 \mathrm{~mL}, 2 \mathrm{mmol})$ was added dropwise with stirring to a mixture of bis-hydrazonoyl chloride $\mathbf{1}(0.451 \mathrm{~g}, 1 \mathrm{mmol})$ and the appropriate methyl arylidene dithiocarbamate $\mathbf{2 a}-\mathbf{d}, \mathbf{6} \mathbf{a}-\mathbf{c}$ and $\mathbf{8 a} \mathbf{a} \mathbf{b}$ $(2 \mathrm{mmol})$ in ethanol $(20 \mathrm{~mL})$ for $30 \mathrm{~min}$. The resulting solid product was collected and recrystallized from DMF to give the corresponding products $5 \mathbf{a}-\mathbf{d}, 7 \mathbf{a}-\mathbf{c}$ and $\mathbf{9 a}, \mathbf{b}$, respectively.

Diethyl 4,4'-(biphenyl-4,4'-diyl)bis(5-(benzylidenehydrazono)-4,5-dihydro-1,3,4-thiadiazole-2-carboxylate) (5a). Yellow solid, (73\% yield), m.p. 194-196 ${ }^{\circ} \mathrm{C}$; IR (KBr) $v_{\max }$ 3032, $2964(\mathrm{C}-\mathrm{H}), 1730(\mathrm{C}=\mathrm{O}), 1609(\mathrm{C}=\mathrm{N})$ $\mathrm{cm}^{-1} ;{ }^{1} \mathrm{H}-\mathrm{NMR}\left(\mathrm{DMSO}-d_{6}\right) \delta 1.32\left(\mathrm{t}, 6 \mathrm{H}, J=7.2 \mathrm{~Hz}, 2 \mathrm{CH}_{2} \mathrm{CH}_{3}\right), 4.35\left(\mathrm{q}, 4 \mathrm{H}, J=7.2 \mathrm{~Hz}, 2 \mathrm{CH}_{2} \mathrm{CH}_{3}\right)$, 7.47-7.88 (m, 14H, Ar-H), $8.04(\mathrm{~d}, 4 \mathrm{H}, J=7.2 \mathrm{~Hz}, \mathrm{Ar}-\mathrm{H}) ; 8.47(\mathrm{~s}, 2 \mathrm{H}, 2 \mathrm{CH}=\mathrm{N}) ;{ }^{13} \mathrm{C}-\mathrm{NMR}\left(\mathrm{DMSO}-d_{6}\right)$

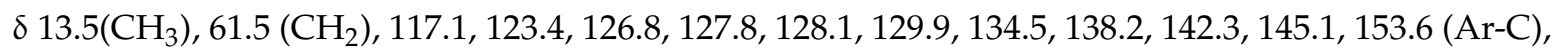
$162.5(\mathrm{C}=\mathrm{O})$. Anal. Calcd. for $\mathrm{C}_{36} \mathrm{H}_{30} \mathrm{~N}_{8} \mathrm{O}_{4} \mathrm{~S}_{2}$ (702.80): $\mathrm{C}, 61.52 ; \mathrm{H}, 4.30 ; \mathrm{N}, 15.94$. Found: $\mathrm{C}, 61.38 ; \mathrm{H}$, $4.19 ; \mathrm{N}, 15.77 \%$.

Diethyl 4,4'-(biphenyl-4,4'-diyl)bis(5-((4-methylbenzylidene)hydrazono)-4,5-dihydro-1,3,4-thiadiazole-2 -carboxylate) (5b). Yellow solid, (75\% yield), m.p. 186-188 ${ }^{\circ} \mathrm{C}$; IR (KBr) $v_{\max } 3049,2974(\mathrm{C}-\mathrm{H}), 1725$ $(\mathrm{C}=\mathrm{O}), 1610(\mathrm{C}=\mathrm{N}) \mathrm{cm}^{-1},{ }^{1} \mathrm{H}-\mathrm{NMR}\left(\mathrm{DMSO}-d_{6}\right) \delta 1.29\left(\mathrm{t}, 6 \mathrm{H}, J=6.9 \mathrm{~Hz}, 2 \mathrm{CH}_{2} \mathrm{CH}_{3}\right), 2.35\left(\mathrm{~s}, 6 \mathrm{H}, 2 \mathrm{CH}_{3}\right)$, $4.34\left(\mathrm{q}, 4 \mathrm{H}, J=6.9 \mathrm{~Hz}, 2 \mathrm{CH}_{2} \mathrm{CH}_{3}\right), 7.21(\mathrm{~d}, 4 \mathrm{H}, J=7.5 \mathrm{~Hz}, \mathrm{Ar}-\mathrm{H}), 7.66(\mathrm{~d}, 4 \mathrm{H}, J=7.5 \mathrm{~Hz}, \mathrm{Ar}-\mathrm{H}), 7.84(\mathrm{~d}$, $4 \mathrm{H}, J=8.7 \mathrm{~Hz}, \mathrm{Ar}-\mathrm{H}), 8.05(\mathrm{~d}, 4 \mathrm{H}, J=8.7 \mathrm{~Hz}, \mathrm{Ar}-\mathrm{H}), 8.43(\mathrm{~s}, 2 \mathrm{H}, 2 \mathrm{CH}=\mathrm{N}) ;{ }^{13} \mathrm{C}-\mathrm{NMR}$ (DMSO-d $\left.{ }_{6}\right) \delta 14.4$, $22.3\left(2 \mathrm{CH}_{3}\right), 61.8\left(\mathrm{CH}_{2}\right), 115.5,122.7,126.9,127.3,128.9,131.2,139.0,139.7,146.5,147.8,153.4$ (Ar-C), $163.1(\mathrm{C}=\mathrm{O}) ; \mathrm{MS} m / z(\%) 730\left(\mathrm{M}^{+}, 4\right), 627(9), 457$ (26), 226 (41), 119 (100), 87 (73), 59 (69). Anal. Calcd. for $\mathrm{C}_{38} \mathrm{H}_{34} \mathrm{~N}_{8} \mathrm{O}_{4} \mathrm{~S}_{2}$ (730.86): C, 62.45; H, 4.69; N, 15.33. Found: $\mathrm{C}, 62.36 ; \mathrm{H}, 4.60 ; \mathrm{N}, 15.24 \%$.

Diethyl 4,4'-(biphenyl-4,4'-diyl)bis(5-((thiophen-2-ylmethylene)hydrazono)-4,5-dihydro-1,3,4-thiadiazole2-carboxylate) (5c). Yellow solid, (74\% yield), m.p. 251-253 ${ }^{\circ} \mathrm{C}$; IR (KBr) $v_{\max } 3041,2980$ (C-H), 1733 $(\mathrm{C}=\mathrm{O}), 1598(\mathrm{C}=\mathrm{N}) \mathrm{cm}^{-1}$; ${ }^{1} \mathrm{H}-\mathrm{NMR}\left(\mathrm{DMSO}-d_{6}\right) \delta 1.31\left(\mathrm{t}, 6 \mathrm{H}, J=6.9 \mathrm{~Hz}, 2 \mathrm{CH}_{2} \mathrm{CH}_{3}\right), 4.34(\mathrm{q}, 4 \mathrm{H}$, $\left.J=7.2 \mathrm{~Hz}, 2 \mathrm{CH}_{2} \mathrm{CH}_{3}\right), 7.14-7.73(\mathrm{~m}, 6 \mathrm{H}, \mathrm{Ar}-\mathrm{H}), 7.84(\mathrm{~d}, 4 \mathrm{H}, J=8.4 \mathrm{~Hz}, \mathrm{Ar}-\mathrm{H}), 8.07(\mathrm{~d}, 4 \mathrm{H}, J=8.4 \mathrm{~Hz}$, Ar-H), $8.61(\mathrm{~s}, 2 \mathrm{H}, 2 \mathrm{CH}=\mathrm{N}) ;{ }^{13} \mathrm{C}-\mathrm{NMR}\left(\mathrm{DMSO}-d_{6}\right) \delta 14.4\left(\mathrm{CH}_{3}\right), 62.7\left(\mathrm{CH}_{2}\right), 115.5,123.4,127.0,128.0$, 130.4, 132.4, 133.3, 139.1, 143.1, 158.3, 159.8 (Ar-C), 165.0 (C=O); MS m/z (\%) $714\left(\mathrm{M}^{+}, 7\right), 542$ (16), 364 (45), 171 (83), 109 (100), 62 (68). Anal. Calcd. for $\mathrm{C}_{32} \mathrm{H}_{26} \mathrm{~N}_{8} \mathrm{O}_{4} \mathrm{~S}_{4}$ (714.86): C, 53.76; H, 3.67; N, 15.67. Found: C, 53.69; H, 3.50; N, 15.43\%.

Diethyl 4,4'-(biphenyl-4,4'-diyl)bis(5-((furan-2-ylmethylene)hydrazono)-4,5-dihydro-1,3,4-thiadiazole2-carboxylate) (5d). Yellow solid, (70\% yield), m.p. 283-285 ${ }^{\circ} \mathrm{C}$; IR (KBr) $v_{\max } 3050,2973(\mathrm{C}-\mathrm{H}), 1732$ $(\mathrm{C}=\mathrm{O}), 1616(\mathrm{C}=\mathrm{N}) \mathrm{cm}^{-1}{ }^{1} \mathrm{H}-\mathrm{NMR}\left(\mathrm{DMSO}-\mathrm{d}_{6}\right) \delta 1.32\left(\mathrm{t}, 6 \mathrm{H}, J=7.2 \mathrm{~Hz}, 2 \mathrm{CH}_{2} \mathrm{CH}_{3}\right), 4.35(\mathrm{q}, 4 \mathrm{H}$, $\left.J=7.2 \mathrm{~Hz}, 2 \mathrm{CH}_{2} \mathrm{CH}_{3}\right), 7.08-7.71(\mathrm{~m}, 6 \mathrm{H}, \mathrm{Ar}-\mathrm{H}), 7.87(\mathrm{~d}, 4 \mathrm{H}, J=8.4 \mathrm{~Hz}, \mathrm{Ar}-\mathrm{H}), 8.05(\mathrm{~d}, 4 \mathrm{H}, J=8.4 \mathrm{~Hz}$, Ar-H), $8.62(\mathrm{~s}, 2 \mathrm{H}, 2 \mathrm{CH}=\mathrm{N})$; MS $m / z(\%) 682\left(\mathrm{M}^{+}, 3\right), 368$ (16), 224 (29), 152 (35), 80 (100), 52 (84). Anal. Calcd. for $\mathrm{C}_{32} \mathrm{H}_{26} \mathrm{~N}_{8} \mathrm{O}_{6} \mathrm{~S}_{2}$ (682.73): $\mathrm{C}, 56.30 ; \mathrm{H}, 3.84 ; \mathrm{N}, 16.41$. Found: $\mathrm{C}, 56.17 ; \mathrm{H}, 3.81 ; \mathrm{N}, 16.28 \%$.

Diethyl 4,4'-(biphenyl-4,4'-diyl)bis(5-((1-(naphthalen-2-yl)ethylidene)hydrazono)-4,5-dihydro-1,3,4-thiadiazole -2-carboxylate) (7a). Yellow solid, (72\% yield), m.p. 243-245 ${ }^{\circ} \mathrm{C}$; IR (KBr) $v_{\max } 3046,2924(\mathrm{C}-\mathrm{H}), 1718$ $(\mathrm{C}=\mathrm{O}), 1599(\mathrm{C}=\mathrm{N}) \mathrm{cm}^{-1} ;{ }^{1} \mathrm{H}-\mathrm{NMR}\left(\mathrm{DMSO}-d_{6}\right) \delta 1.32\left(\mathrm{t}, 6 \mathrm{H}, J=7.2 \mathrm{~Hz}, 2 \mathrm{CH}_{2} \mathrm{CH}_{3}\right), 2.49\left(\mathrm{~s}, 6 \mathrm{H}, 2 \mathrm{CH}_{3}\right)$, 4.35 (q, $\left.4 \mathrm{H}, J=7.2 \mathrm{~Hz}, 2 \mathrm{CH}_{2} \mathrm{CH}_{3}\right), 7.46-8.19$ (m, 20H, Ar-H), 8.34 (s, 2H, Naphthalene-H1); MS m/z (\%) $830\left(\mathrm{M}^{+}, 17\right), 378$ (88), 263 (29), 177 (48), 127 (100), 63 (71). Anal. Calcd. for $\mathrm{C}_{46} \mathrm{H}_{38} \mathrm{~N}_{8} \mathrm{O}_{4} \mathrm{~S}_{2}$ (830.98): C, 66.49; H, 4.61; N, 13.48. Found: C, 66.36; H, 4.49; N, 13.41\%. 
Diethyl 4,4'-(biphenyl-4,4'-diyl)bis(5-((1-(furan-2-yl)ethylidene)hydrazono)-4,5-dihydro-1,3,4-thiadiazole-2 -carboxylate) (7b). Yellow solid, (73\% yield), m.p. 259-261 ${ }^{\circ} \mathrm{C}$; IR (KBr) v $\max 3055,2978,2928$ (C-H), $1715(\mathrm{C}=\mathrm{O}), 1601(\mathrm{C}=\mathrm{N}) \mathrm{cm}^{-1},{ }^{1} \mathrm{H}-\mathrm{NMR}\left(\mathrm{DMSO}-d_{6}\right) \delta 1.34\left(\mathrm{t}, 6 \mathrm{H}, J=7.2 \mathrm{~Hz}, 2 \mathrm{CH}_{2} \mathrm{CH}_{3}\right), 2.32(\mathrm{~s}, 6 \mathrm{H}$, $\left.2 \mathrm{CH}_{3}\right), 4.35\left(\mathrm{q}, 4 \mathrm{H}, \mathrm{J}=7.2 \mathrm{~Hz}, 2 \mathrm{CH}_{2} \mathrm{CH}_{3}\right), 6.63(\mathrm{~d}, 2 \mathrm{H}, J=2.7 \mathrm{~Hz}), 7.02(\mathrm{~d}, 2 \mathrm{H}, J=2.7 \mathrm{~Hz}), 7.39(\mathrm{~m}, 2 \mathrm{H})$, $7.87(\mathrm{~d}, 4 \mathrm{H}, \mathrm{J}=9.0 \mathrm{~Hz}, \mathrm{Ar}-\mathrm{H}), 8.05(\mathrm{~d}, 4 \mathrm{H}, \mathrm{J}=9.0 \mathrm{~Hz}, \mathrm{Ar}-\mathrm{H}) ;{ }^{13} \mathrm{C}-\mathrm{NMR}\left(\mathrm{DMSO}-d_{6}\right) \delta 14.4,15.1\left(2 \mathrm{CH}_{3}\right)$, $62.3\left(\mathrm{CH}_{2}\right), 116.5,122.6,127.9,128.3,128.9,133.2,138.0,142.7,146.7,153.0,157.8(\mathrm{Ar}-\mathrm{C}), 163.4(\mathrm{C}=\mathrm{O})$; MS m/z (\%) $710\left(\mathrm{M}^{+}, 20\right), 447$ (22), 226 (29), 109 (52), 59 (100). Anal. Calcd. for $\mathrm{C}_{34} \mathrm{H}_{30} \mathrm{~N}_{8} \mathrm{O}_{6} \mathrm{~S}_{2}$ (710.78): C, 57.45; H, 4.25; N, 15.76. Found: C, 57.32; H, 4.21; N, 15.53\%.

Diethyl 4,4'-(biphenyl-4,4'-diyl)bis(5-((1-(pyridin-3-yl)ethylidene)hydrazono)-4,5-dihydro-1,3,4-thiadiazole-2carboxylate) (7c). Yellow solid, (70\% yield), m.p. 263-265 ${ }^{\circ} \mathrm{C}$; IR (KBr) v max 3059, 2922 (C-H), 1736 $(\mathrm{C}=\mathrm{O}), 1599(\mathrm{C}=\mathrm{N}) \mathrm{cm}^{-1} ;{ }^{1} \mathrm{H}-\mathrm{NMR}\left(\mathrm{DMSO}-d_{6}\right) \delta 1.31\left(\mathrm{t}, 6 \mathrm{H}, J=7.2 \mathrm{~Hz}, 2 \mathrm{CH}_{2} \mathrm{CH}_{3}\right), 2.49\left(\mathrm{~s}, 6 \mathrm{H}, 2 \mathrm{CH}_{3}\right)$, $4.32\left(\mathrm{q}, 4 \mathrm{H}, \mathrm{J}=7.2 \mathrm{~Hz}, 2 \mathrm{CH}_{2} \mathrm{CH}_{3}\right), 7.48-7.75$ (m, 2H, Pyridine-H), $7.84(\mathrm{~d}, 4 \mathrm{H}, J=8.4 \mathrm{~Hz}, \mathrm{Ar}-\mathrm{H}), 8.07(\mathrm{~d}$, $4 \mathrm{H}, \mathrm{J}=8.4 \mathrm{~Hz}, \mathrm{Ar}-\mathrm{H}), 8.29-9.05$ (m, 6H, Pyridine-H); ${ }^{13} \mathrm{C}-\mathrm{NMR}$ (DMSO-d $)_{6} \delta 14.6,15.3\left(2 \mathrm{CH}_{3}\right), 62.6$ $\left(\mathrm{CH}_{2}\right), 114.2,115.5,120.5,127.3,128.0,130.8,132.2,134.2,142.1,143.5,152.6,159.8$ (Ar-C), $167.1(\mathrm{C}=\mathrm{O})$; MS m/z (\%): $732\left(\mathrm{M}^{+}, 3\right), 415$ (24), 276 (37), 205 (64), 152 (60), 84 (100). Anal. Calcd. for $\mathrm{C}_{36} \mathrm{H}_{32} \mathrm{~N}_{10} \mathrm{O}_{4} \mathrm{~S}_{2}$ (732.83): C, 59.00; H, 4.40; N, 19.11 Found: C, 58.86; H, 4.31; N, 19.02\%.

Diethyl 4,4'-(biphenyl-4,4'-diyl)bis(5-(cyclohexylidenehydrazono)-4,5-dihydro-1,3,4-thiadiazole-2-carboxylate) (9a). Yellow solid, (72\% yield), m.p. 233-235 ${ }^{\circ} \mathrm{C}$; IR (KBr) $v_{\max }$ 3032, 2929 (C-H), $1716(\mathrm{C}=\mathrm{O}), 1616$ $(\mathrm{C}=\mathrm{N}) \mathrm{cm}^{-1} ;{ }^{1} \mathrm{H}-\mathrm{NMR}\left(\mathrm{DMSO}-d_{6}\right) \delta 1.34\left(\mathrm{t}, 6 \mathrm{H}, J=7.2 \mathrm{~Hz}, 2 \mathrm{CH}_{2} \mathrm{CH}_{3}\right), 1.61\left(\mathrm{~m}, 12 \mathrm{H}, 6 \mathrm{CH}_{2}\right), 2.34(\mathrm{~m}$, $\left.4 \mathrm{H}, 2 \mathrm{CH}_{2}\right), 2.63\left(\mathrm{~m}, 4 \mathrm{H}, 2 \mathrm{CH}_{2}\right), 4.38\left(\mathrm{q}, 4 \mathrm{H}, J=7.2 \mathrm{~Hz}_{2} \mathrm{CH}_{2} \mathrm{CH}_{3}\right), 7.88(\mathrm{~d}, 4 \mathrm{H}, J=8.4 \mathrm{~Hz}, \mathrm{Ar}-\mathrm{H}), 8.04$ $(\mathrm{d}, 4 \mathrm{H}, J=8.4 \mathrm{~Hz}, \mathrm{Ar}-\mathrm{H}) ;{ }^{13} \mathrm{C}-\mathrm{NMR}\left(\mathrm{DMSO}-d_{6}\right) \delta 14.4\left(\mathrm{CH}_{3}\right), 25.0,27.5,35.2,63.4\left(\mathrm{CH}_{2}\right), 116.1,122.6$, 126.2, 133.6, 137.2, 146.5, 160.0 (Ar-C), 163.1(C=O); MS m/z (\%): $686\left(\mathrm{M}^{+}, 6\right), 415(16), 226(24), 194(52)$, 104 (79), 90 (100). Anal. Calcd. for $\mathrm{C}_{34} \mathrm{H}_{38} \mathrm{~N}_{8} \mathrm{O}_{4} \mathrm{~S}_{2}$ (686.85): C, 59.45; H, 5.58; N, 16.31. Found: C, 59.25; H, 5.39; N, $16.22 \%$.

Ethyl 4-(4'-((Z)-5-(ethoxycarbonyl)-2-((2-oxoindolin-3-ylidene)hydrazono)-1,3,4-thiadiazol-3(2H)-yl)biphenyl -4-yl)-5-((Z)-(2-oxoindolin-3-ylidene)hydrazono)-4,5-dihydro-1,3,4-thiadiazole-2-carboxylate (9b). Yellow solid, (67\% yield), m.p. 266-268 ${ }^{\circ} \mathrm{C}$; IR (KBr) $v_{\max } 3344(\mathrm{NH}), 3033,2978(\mathrm{C}-\mathrm{H}), 1724,1681(2 \mathrm{C}=\mathrm{O}), 1607$ $(\mathrm{C}=\mathrm{N}) \mathrm{cm}^{-1} ;{ }^{1} \mathrm{H}-\mathrm{NMR}\left(\mathrm{DMSO}-d_{6}\right) \delta 1.34\left(\mathrm{t}, 6 \mathrm{H}, \mathrm{J}=6.9 \mathrm{~Hz}, 2 \mathrm{CH}_{2} \mathrm{CH}_{3}\right), 4.42\left(\mathrm{q}, 4 \mathrm{H}, \mathrm{J}=6.9 \mathrm{~Hz}, 2 \mathrm{CH}_{2} \mathrm{CH}_{3}\right)$, 6.85-8.01 (m, 16H, Ar-H), 10.65 (s, br, 2H, 2NH, D $2 \mathrm{O}-$ exchangeable); ${ }^{13} \mathrm{C}-\mathrm{NMR}$ (DMSO-d 6$) \delta 14.3\left(\mathrm{CH}_{3}\right)$, $62.6\left(\mathrm{CH}_{2}\right), 114.2,115.5,117.5,120.5,123.5,124.1,126.9,127.3,130.8,131.2,134.2,142.1,159.8$ (Ar-C), 163.4, $170.6(2 \mathrm{C}=\mathrm{O})$; MS m/z (\%): $784\left(\mathrm{M}^{+}, 4\right), 599(18), 392(26), 257$ (38), 166 (68), 70 (100), 58 (42). Anal. Calcd. for $\mathrm{C}_{38} \mathrm{H}_{28} \mathrm{~N}_{10} \mathrm{O}_{6} \mathrm{~S}_{2}$ (784.82): C, 58.15; H, 3.60; N, 17.85. Found: C, 58.08; H, 3.47; N, $17.69 \%$.

\subsection{Pharmacology}

Anticancer Activity

The cytotoxic evaluation of the synthesized compounds was carried out at the Regional Center for Mycology and Biotechnology at Al-Azhar University, Cairo, Egypt according to the reported method [29].

\section{Conclusions}

Various bis(1,3,4-thiadiazole) derivatives were synthesized in efficient and easy protocol. Biological studies revealed that bis-thiadiazole derivatives $\mathbf{5 c}, \mathbf{d}, \mathbf{7 b}, \mathbf{c}$ and $\mathbf{9 b}$ had higher antitumor activity than the standard drug Imitanib.

Acknowledgments: The authors extend their sincere appreciation to the Deanship of Scientific Research at the King Saud University for its funding this Prolific Research group (PRG-1437-29).

Author Contributions: A.O.A., S.M.G. and N.A.K. designed research; A.O.A., S.M.G., N.A.K. and Y.N.M. performed research, analyzed the data, wrote the paper and approved the final manuscript. 
Conflicts of Interest: The authors declare that there is no conflict of interests regarding the publication of this paper.

\section{References}

1. Hu, Y.; Li, C.Y.; Wang, X.M.; Yang, Y.H.; Zhu, H.L. 1,3,4-Thiadiazole: Synthesis, reactions, and applications in medicinal, agricultural, and materials chemistry. Chem. Rev. 2014, 114, 5572-5610. [CrossRef] [PubMed]

2. Yar, M.S.; Akhter, M.W. Synthesis and anticonvulsant activity of substituted oxadiazole and thiadiazole derivatives. Acta Pol. Pharm. 2009, 66, 393-397. [PubMed]

3. Azam, F.; Ibn-Rajab, I.A.; Alruiad, A.A. Adenosine $\mathrm{A}_{2 \mathrm{~A}}$ receptor antagonists as novel anti-parkinsonian agents: a review of structure-activity relationships. Die Pharm. 2009, 64, 771-775. [CrossRef]

4. Lalezari, I.; Shafiee, A.; Badaly, A.; Salimi, M.M.; Khoyi, M.A.; Abtahi, F.; Zarrindast, M.R. Synthesis and pharmacological activity of 5-substituted 2-(N,N-dialkylaminoethyl)amino- and 2- $N$-methyl piperazinyl-1,3,4-thiadiazoles. J. Pharm. Sci. 1975, 64, 1250-1252. [CrossRef] [PubMed]

5. Bhattacharya, P.; Leonard, J.T.; Roy, K. Exploring QSAR of thiazole and thiadiazole derivatives as potent and selective human adenosine A3 receptor antagonists using FA and GFA techniques. Bioorg. Med. Chem. 2005, 15, 1159-1165. [CrossRef] [PubMed]

6. Zhao, J.; Xuan, L.; Zhao, H.; Cheng, J.; Fu, X.; Li, S.; Jing, F.; Liu, Y.; Chen, B. Synthesis and antitumor activities of 1,3,4-thiadiazole derivatives possessing benzisoselenazolone scaffold. Chem. Res. Chin. Univ. 2014, 30, 764-769. [CrossRef]

7. Kumar, D.; Kumar, N.M.; Chang, K.-H.; Shah, K. Synthesis and anticancer activity of 5-(3-indolyl)-1,3,4-thiadiazoles. Eur. J. Med. Chem. 2010, 45, 4664-4668. [CrossRef] [PubMed]

8. Mathew, V.; Keshavayya, J.; Vaidya, V.P.; Giles, D. Studies on synthesis and pharmacological activities of 3,6-disubstituted-1,2,4-triazolo[3,4-b]-1,3,4-thiadiazoles and their dihydro analogues. Eur. J. Med. Chem. 2007, 42, 823-840. [CrossRef] [PubMed]

9. Noolvi, M.N.; Patel, H.M.; Kamboj, S.; Cameotra, S.S. Synthesis and antimicrobial evaluation of novel 1,3,4-thiadiazole derivatives of 2-(4-formyl-2-methoxyphenoxy) acetic acid. Arab. J Chem. 2012. [CrossRef]

10. Chawla, A.; Chawla, P.; Baghel, U.S. Synthesis and antimicrobial evaluation of 5-aryl-1,3,4-thiadiazole -2-ylamine derivatives. World J. Pharm. Sci. 2016, 4, 110-114.

11. Karakus, S.; Rollas, S. Synthesis and antituberculosis activity of new N-phenyl-N'-[4-(5-alkyl/arylamino -1,3,4-thiadiazole-2- yl)phenyl]thioureas. Farmaco 2002, 57, 577-581. [CrossRef]

12. Oruc, E.E.; Rollas, S.; Kandermirli, F.; Shvets, N.; Dimoglo, A.S. 1,3,4-Thiadiazole derivatives: Synthesis, structure elucidation, and structure-antituberculosis activity relationship investigation. J. Med. Chem. 2004, 47, 6760-6767. [CrossRef] [PubMed]

13. Foroumadi, A.; Kargar, Z.; Sakhteman, A.; Sharifzadeh, Z.; Feyzmohammadi, R.; Kazemi, M.; Shafiee, A. Synthesis and antimycobacterial activity of some alkyl [5-(nitroaryl)-1,3,4-thiadiazol-2-ylthio]propionates. Bioorg. Med. Chem. Lett. 2006, 16, 1164-1167. [CrossRef] [PubMed]

14. Darwish, E.S.; Kheder, N.A.; Farag, A.M. Synthesis and antimicrobial evaluation of some new pyridine based heterocycles. Heterocycles 2010, 81, 2247-2256.

15. Farag, A.M.; Kheder, N.A.; Mabkhot, Y.N. Synthesis and antimicrobial evaluation of new pyrazole, thiophene, thiazole and 1,3,4-thiadiazole derivatives incorporating pyrimidine ring. Heterocycles 2009, 78, 1787-1798. [CrossRef]

16. Kheder, N.A.; Riyadh, S.M.; Asiry, A.M. Azoles and bis-Azoles: Synthesis and biological evaluation as antimicrobial and anti-cancer agents. Chem. Pharm. Bull. 2013, 61, 504-510. [CrossRef] [PubMed]

17. Gomha, S.M.; Badrey, M.G.; Edrees, M.M. Heterocyclisation of 2,5-diacetyl-3,4-disubstitutedthieno[2,3-b]thiophene bis-thiosemicarbazones leading to bis-thiazoles and bis-1,3,4-thiadiazoles as anti-breast cancer agents. J. Chem. Res. 2016, 120-125. [CrossRef]

18. Dawood, K.M.; Gomha, S.M. Synthesis and anti-cancer activity of 1,3,4-thiadiazole and 1,3-thiazole derivatives having 1,3,4-oxadiazole moiety. J. Heterocycl. Chem. 2015, 52, 1400-1405. [CrossRef]

19. Gomha, S.M.; Riyadh, S.M.; Mahmmoud, E.A.; Elaasser, M.M. Synthesis and anticancer activity of arylazothiazoles and 1,3,4-thiadiazoles using chitosan-grafted-poly(4-vinyl pyridine) as a novel copolymer basic catalyst. Heterocycles 2015, 91, 1227-1243. 
20. Gomha, S.M.; Abdelrazek, F.M.; Abdelrahman, A.H.; Metz, P. Synthesis of some novel thiazole, thiadiazole and 1,4-phenylene-bis-thiazole derivatives. Heterocycles 2016, 92, 954-967. [CrossRef]

21. Gomha, S.M.; Abdel-aziz, H.M. Synthesis and antitumor activity of 1,3,4-thiadiazole derivatives bearing coumarine ring. Heterocycles 2015, 91, 583-592. [CrossRef]

22. Gomha, S.M.; Salah, T.A.; Abdelhamid, A.O. Synthesis, characterization and pharmacological evaluation of some novel thiadiazoles and thiazoles incorporating pyrazole moiety as potent anticancer agents. Monatsh. Chem. 2015, 146, 149-158. [CrossRef]

23. Gomha, S.M.; Ahmed, S.A.; Abdelhamid, A.O. Synthesis and cytotoxicity evaluation of some novel thiazoles, thiadiazoles, and pyrido[2,3- $d][1,2,4]$ triazolo[4,3-a]pyrimidin- 5(1H)-one incorporating triazole moiety. Molecules 2015, 20, 1357-1376. [CrossRef] [PubMed]

24. Kheder, N.A.; Darwish, E.S. Diethyl 2,2'-[Biphenyl-4,4'-diyldihydrazin-2-yl-1-ylidene]bis (chloroacetate). Molbank 2014, 2014, M813. [CrossRef]

25. Mahapatra, M.K.; Kulandaivelu, U.; Saiko, P.; Graser, G.; Szekeres, T.; Andrei, G.; Snoeck, R.; Balzarini, J.; Jayaprakash, V. Methyl-2-arylidene hydrazinecarbodithioates: Synthesis and biological activity. Chem. Pap. 2013, 67, 650-656. [CrossRef]

26. Audrieth, L.F.; Scott, E.S.; Kippur, P.S. Hydrazine derivatives of the carbonic and thiocarbonic acids. I. the preparation and properties of thiocarbohydrazide. J. Org. Chem. 1954, 19, 733-741. [CrossRef]

27. Klayman, D.L.; Bartosevich, J.F.; Griffin, T.S.; Mason, C.J.; Scovill, J.P. 2-Acetylpyridine thiosemicarbazones. 1. A new class of potential antimalarial agents. J. Med. Chem. 1979, 22, 855-862. [CrossRef] [PubMed]

28. Scovill, J.P.; Klayman, D.L.; Franchino, C.F. 2-Acetylpyridine thiosemicarbazones. 4. Complexes with transition metals as antimalarial and antileukemic agents. J. Med. Chem. 1982, 25, 1261-1264. [CrossRef] [PubMed]

29. Gomha, S.M.; Edrees, M.M.; El-Arab, E.E. Synthesis and preliminary in vitro cytotoxic evaluation of some novel bis-heterocycles incorporating thienothiophene. J. Heterocycl. Chem. 2016. [CrossRef]

Sample Availability: Samples of the compounds $\mathbf{5 a - d}, \mathbf{7 a - c}$ and $\mathbf{9 a}, \mathbf{b}$ are available from the authors.

(C) 2016 by the authors; licensee MDPI, Basel, Switzerland. This article is an open access article distributed under the terms and conditions of the Creative Commons Attribution (CC-BY) license (http://creativecommons.org/licenses/by/4.0/). 